\title{
satFRET: estimation of Förster resonance energy transfer by acceptor saturation
}

\author{
Martin Beutler · Konstantina Makrogianneli • \\ Rudolf J. Vermeij · Melanie Keppler · Tony Ng • \\ Thomas M. Jovin · Rainer Heintzmann
}

Received: 14 March 2008/Revised: 11 July 2008/Accepted: 21 July 2008/Published online: 4 September 2008

(C) The Author(s) 2008. This article is published with open access at Springerlink.com

\begin{abstract}
We demonstrate theoretically and experimentally the quantification of Förster resonance energy transfer (FRET) by direct and systematic saturation of the excited state of acceptor molecules. This version of acceptor depletion methods for FRET estimation, denoted as "satFRET" is reversible and suitable for time-resolved measurements. The technique was investigated theoretically using the steady-state solution of the differential equation system of donor and acceptor molecular states. The influence of acceptor photobleaching during measurement was included in the model. Experimental verification was achieved with the FRET-pair Alexa 546Alexa 633 loaded on particles in different stoichiometries
\end{abstract}

M. Beutler $(\bowtie)$

Microsensor Research Group,

Max-Planck-Institute for Marine Microbiology,

Celsiusstrasse 1, 28359 Bremen, Germany

e-mail: MartinBeutler@gmx.de

M. Beutler

bionsys, Schwachhauser Heerstr. 85a, 28211 Bremen, Germany

M. Beutler · K. Makrogianneli · M. Keppler · T. Ng ·

R. Heintzmann

King's College London,

Randall Division of Cell and Molecular Biophysics,

Guy's Campus, London SE1 1UL, UK

M. Beutler · R. J. Vermeij - T. M. Jovin · R. Heintzmann

Laboratory of Cellular Dynamics,

Max-Planck-Institute for Biophysical Chemistry,

Am Fassberg 11, 37077 Gottingen, Germany

R. J. Vermeij

Biophysical Engineering Group,

Faculty of Science and Technology, University of Twente,

PO Box 217, 7500AE Enschede, The Netherlands and measured in a confocal microscope. Estimates of energy transfer efficiency by excited state saturation were compared to those obtained by measurements of sensitised emission and acceptor photobleaching. The results lead to a protocol that allows time-resolved FRET measurements of fixed and living cells on a conventional confocal microscope. This procedure was applied to fixed Chinese hamster ovary cells containing a cyan fluorescent protein and yellow fluorescent protein pair. The time resolution of the technique was demonstrated in a live $\mathrm{T}$ cell activation assay comparing the FRET efficiencies measured using a genetically encoded green and red fluorescent protein biosensor for GTP/GDP turnover to those measured by acceptor photobleaching of fixed cells.

Keywords FRET - CFP - YFP - Excited state saturation Fluorescence - Confocal microscopy - Sensitised emission · Photobleaching

$\begin{array}{ll}\text { Abbreviations } & \\ \text { CFP } & \text { Cyan fluorescent protein } \\ \text { GFP } & \text { Green fluorescent protein } \\ \text { RFP } & \text { Red fluorescent protein } \\ \text { YFP } & \text { Yellow fluorescent protein } \\ \text { FRET } & \text { Förster resonance energy transfer } \\ \text { CHO } & \text { Chinese hamster ovary } \\ \text { PBS } & \text { Phosphate buffered saline } \\ \text { saturation FRET } & \text { satFRET. }\end{array}$

\section{Introduction}

Förster resonance energy transfer (FRET) is the method of choice in microscopy, flow cytometry and spectroscopy for 
analysing interactions and/or conformational changes of donor and acceptor molecules (e.g. proteins, ligands and lipids), particularly in living cells. The physical basis of FRET is a distance-dependent quantum mechanical interaction that occurs between chromophores. Exchange of excitation energy between the electronic states of a suitable donor-acceptor FRET pair takes place via a dipole-dipole coupling mechanism. The excited donor fluorophore undergoes deexcitation by transferring energy to the acceptor. This process, however, can only occur if the donor fluorescence emission and the acceptor excitation spectra overlap to a significant degree. The FRET efficiency (relative contribution to the overall deactivation pathways) is dependent on the spectral overlap, the separation and relative orientation of the donor and acceptor molecules, and other physical quantities. FRET is generally operable over the range of $2-10 \mathrm{~nm}$. The involvement of dipole-dipole interactions as the mechanism via which molecules can interact without collisions was first proposed by Perrin (Perrin 1927; Clegg 2006) in a classical description. Later, Förster (1948) built upon Perrin's idea to put forward a theory which provided a quantitative explanation for the non-radiative energy transfer. The use of the transfer mechanism then rapidly evolved to a widely and routinely employed method in molecular and cell biology. Its development has been reviewed and discussed in depth (Andrews and Demidov 1999; Berney and Danuser 2003; Clegg 1996; Jares-Erijman and Jovin 2003, 2006; Marriott and Parker 2003; Stryer 1978; van der Meer 1994), but methodological progress continues to be made.

Due to the sixth power law dependence on distance of the FRET process, it can be employed as a very sensitive molecular ruler [(Clegg 1996; Stryer 1978); for a detailed single-molecule re-examination of the classical experiment of Stryer and Haugland (1967) see (Schuler et al. 2005)].

Many FRET determinations are based on spectral methods that involve the measurement of sensitised acceptor emission (Jares-Erijman and Jovin 2006; Lakowicz 2006). They can be used by themselves but may also be combined with any of the other approaches described below (Elangovan et al. 2003). In the case of acceptors with large Stokes shifts (separation of excitation and emission bands), the donor fluorescence can be monitored by excitation at its characteristic absorption region and measurement of the emission in both the donor and acceptor emission bands. Similarly, large donor Stokes shifts can help to avoid direct excitation of the acceptor at the donor excitation wavelength. However, many FRET pairs that are suitable for biological experiments (e.g. green fluorescent protein and its spectral derivatives) exhibit substantial spectral overlap (Elangovan et al. 2003). In other words, the donor also fluoresces in the wavelength range of the acceptor emission channel (leading to "donor crosstalk") and the acceptor may also be directly excited in the donor absorption band. These effects can be corrected mathematically ("spectral unmixing") by careful calibration based on the properties of the respective individual fluorophores (Neher and Neher 2004; Wlodarczyk et al. 2008; Zimmermann et al. 2002). A problem occurs in FRET experiments if not all of the donors are bound to one or more acceptors, such that free donors are present that do not exhibit FRET although fluorescence is detected in both detection channels. In this case, additional measurements are required in combination with sensitised emission to achieve reasonable estimates of the concentration of the two species and the FRET-efficiency of the interaction (Clegg 1996; Elangovan et al. 2003; Hoppe et al. 2002; Wlodarczyk et al. 2008; Hoppe et al. 2008).

Another popular method generates an internal reference state by photodestruction of the acceptor (acceptor photobleaching FRET). Problematic issues for these methods include incomplete bleaching of the acceptors, residual absorption of bleached acceptors at the donor emission wavelengths, photodestruction of donors during acceptor depletion and generation of fluorescent photoproducts (Valentin et al. 2005). One obvious disadvantage of the approaches based on irreversible photodestruction is that the experiment can be performed only once at a given location. In addition, extensive photobleaching is often lethal to live cells. Thus, these techniques are not well suited for prolonged observation, prompting the development of alternative reversible strategies for "acceptor depletion" (Jares-Erijman and Jovin 2003, 2006).

Fluorescence lifetime imaging microscopy [FLIM; (Suhling et al. 2005)] is a rapid, robust and non-destructive method for FRET estimation (Bunt and Wouters 2004). FRET efficiencies and the fractional concentration of FRET pairs can be estimated, for example with spectralresolved FLIM (Clayton et al. 2002, 2004; Colyer et al. 2007; Digman et al. 2008; Hanley et al. 2002; Lakowicz 2006; Redford and Clegg 2005).

In this paper a new approach was tested that is based on excited state saturation of the acceptor molecules ["frustrated FRET" method designated Ie3 in (Jares-Erijman and Jovin 2003)]. It has time-resolved measuring capabilities and the potential to be combined with other existing methods.

Donor or acceptor molecules can be driven into excited state saturation by intense excitation light (Hanninen et al. 1996; Jares-Erijman and Jovin 2003). Davis et al. (2005) have modelled and used saturation effects in fluorescence correlation spectroscopy.

At low light intensities, the fluorescence emission of a fluorophore population is proportional to the excitation 
intensity. When exposed to higher excitation light intensities, an increasing fraction of the fluorophore is driven into the excited state. At saturation, the fluorescence intensity reaches a limit defined solely by the intrinsic radiative rate (Jares-Erijman and Jovin 2003). In this limiting case, re-excitation is virtually instantaneous after fluorescence emission. At less extreme intensities, the proportionality between excitation intensity and fluorescence emission is violated because many molecules have a high probability of already being excited at the instant of excitation, e.g. during a light pulse. We apply these principles to the acceptor of a FRET system, noting that in most chemical systems, acceptors excited directly into saturation will no longer absorb in the spectral range required for FRET. Under these conditions, the donor will be de-quenched since the spectral overlap required for FRET is no longer satisfied. Saturation FRET (satFRET) is based on the systematic exploitation of this phenomenon.

To establish and test the method, the system of differential equations describing the molecular states and transitions of donor and acceptor molecules was solved for the steady-state situation. The influence of acceptor photobleaching on the efficiency estimates caused by the intense acceptor excitation required for achieving saturation of the molecules was investigated. A photobleaching correction function was introduced and applied to the situation of most biosensors with a fixed donor-toacceptor-ratio of unity. The effect of saturating acceptor light intensities was simulated and investigated experimentally in a confocal microscope with FRET pairs placed on the surface of particles. FRET efficiencies could be measured by systematically employing acceptor excited state saturation. Spectral unmixing was also feasible. The results were compared to those obtained conventionally from donor and sensitised emission intensities. Finally, a fast measuring protocol allowing time-resolved measurements was conceived and applied to fixed and living cells.

\section{Methods}

\section{Molecular state models}

The system of differential equations applying to the transitions in a FRET pair consisting of one donor and one acceptor as given in Fig. 1.

The system of equations can be solved for the steadystate condition (all four derivatives set to 0 and the conservation condition: $D A+D^{*} A+D A^{*}+D^{*} A^{*}=1$ ), yielding for the acceptor excited $S$-state population:
(A)

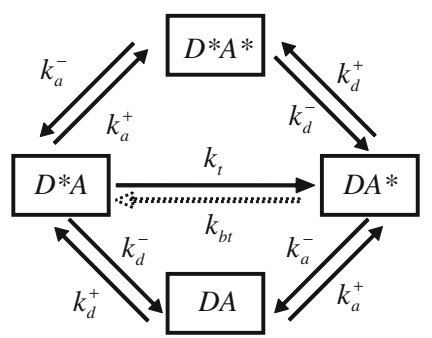

(B)

$\frac{d}{d t}\left(\begin{array}{c}D A(t) \\ D^{*} A(t) \\ D A^{*}(t) \\ D^{*} A^{*}(t)\end{array}\right)=\left(\begin{array}{cccc}-\left(k_{d}^{+}+k_{a}^{+}\right) & k_{d}^{-} & k_{a}^{-} & 0 \\ k_{d}^{+} & -\left(k_{d}^{-}+k_{a}^{+}+k_{t}\right) & 0 & k_{a}^{-} \\ k_{a}^{+} & k_{t} & -\left(k_{a}^{-}+k_{d}^{+}\right) & k_{d}^{-} \\ 0 & k_{a}^{+} & k_{d}^{+} & -\left(k_{a}^{-}+k_{d}^{-}\right)\end{array}\right)\left(\begin{array}{c}D A(t) \\ D^{*} A(t) \\ D A^{*}(t) \\ D^{*} A^{*}(t)\end{array}\right)$

Fig. 1 a State diagram of the donor-acceptor system (DA). Asterisks indicate an excited singlet state of the donor $\left(D^{*}\right)$ and/or acceptor $\left(A^{*}\right) . \quad k \mathrm{~s}$ are rate constants. Before light absorption, the donor molecule is in its ground state, denoted as $D$. By absorption of a photon $\left(k_{d}^{+}\right)$it passes to the first excited singlet state $D^{*}$. From there it can deactivate via light emission (fluorescence) and non-radiative means (combined rate, $k_{d}^{-}=\tau_{d}^{-1}$ ) and, if present, FRET-mediated indirect excitation of the acceptor $\left(k_{t}\right)$. FRET shortens the lifetime and decreases the quantum yield of the donor with an efficiency of $E_{0}=$ $k_{t} /\left(k_{d}^{-}+k_{t}\right)$, when the acceptor is mostly in the ground state. The transfer process is partially reversible under some circumstances, particularly when $D$ and $A$ are similar molecules; we do not include back-transfer in our treatment. The acceptor excited state $A^{*}$ is generated either by direct excitation $\left(k_{a}^{+}=k_{a}^{+}\left(\lambda_{D, \mathrm{ex}}\right)+k_{a}^{+}\left(\lambda_{A, \mathrm{ex}}\right)\right.$ with $\left(k_{a}^{+}\left(\lambda_{D, \mathrm{ex}}\right)=\right.$ acceptor excitation rate by donor excitation light and $k_{a}^{+}\left(\lambda_{A, \mathrm{ex}}\right)=$ acceptor excitation by acceptor light) or via FRET $\left(k_{t}\right) . A^{*}$ de-excites by light-emission (if fluorescent) and/or nonradiative pathways (combined rate $k_{a}^{-}=\tau_{a}^{-1}$ ). b Time dependent mathematical description corresponding to (a), see also (Hanninen et al. 1996)

$$
\begin{aligned}
& A^{*}=D A^{*}+D^{*} A^{*}= \\
& \frac{k_{a}^{+}\left(k_{d}^{-}+k_{d}^{+}\right) k_{\mathrm{sum}}+\left(k_{a}^{+}+k_{d}^{+}\right)\left(k_{a}^{-}+k_{d}^{-}+k_{d}^{+}\right) k_{t}}{\left(k_{a}^{-}+k_{a}^{+}\right)\left(k_{d}^{-}+k_{d}^{+}\right) k_{\mathrm{sum}}+\left[\left(k_{a}^{+}+k_{d}^{+}\right)\left(k_{d}^{-}+k_{d}^{+}\right)+k_{a}^{-} k_{\mathrm{sum}}\right] k_{t}}
\end{aligned}
$$

with $k_{\text {sum }}=k_{a}^{-}+k_{a}^{+}+k_{d}^{-}+k_{d}^{+}$. The excitation of a state is denoted by a star in superscript. A superscript "+" means an excitation rate and "-" means a deexcitation rate. " $a$ " and " $d$ " as a subscript on the rate constants refers to donor and acceptor, respectively.

The steady-state acceptor fluorescence $F_{A}$ is given by

$F_{A} \propto k_{f, a} A^{*}$

with $k_{f, a}$ the intrinsic acceptor radiative rate constant.

Similarly, for the donor S-state population:

$$
\begin{aligned}
& D^{*}=D^{*} A+D^{*} A^{*}= \\
& \frac{\left(k_{a}^{-}+k_{a}^{+}\right) k_{d}^{+} k_{\mathrm{sum}}+k_{d}^{+}\left(k_{a}^{+}+k_{d}^{+}\right) k_{t}}{\left(k_{a}^{-}+k_{a}^{+}\right)\left(k_{d}^{-}+k_{d}^{+}\right) k_{\mathrm{sum}}+\left[\left(k_{a}^{+}+k_{d}^{+}\right)\left(k_{d}^{-}+k_{d}^{+}\right)+k_{a}^{-} k_{\mathrm{sum}}\right] k_{t}}
\end{aligned}
$$


with the corresponding steady-state donor fluorescence:

$F_{D} \propto k_{f, d} D^{*}$

and $k_{f, d}$ the intrinsic donor radiative rate.

\section{Cover slip coating}

The cover slips were coated by Cell-Tak [BD, US, (Allen et al. 1992; Jelle Eygensteyn et al. 1997)] using the adsorption technique (Allen et al. 1992; Jelle Eygensteyn et al. 1997). The method is based on the observation that Cell-Tak comes out of solution as the $\mathrm{pH}$ is raised and spontaneously adsorbs to the first surface it contacts. The resulting coating is quite thin (probably close to a protein mono-layer) and more uniform than that achieved by other methods.

\section{Bead preparation}

A suspension of commercially available biotin-coated polystyrene beads (Invitrogen, diameter $1 \mu \mathrm{m}, \mathrm{F} 8769$ ) was diluted tenfold with phosphate buffered saline (PBS), $\mathrm{pH} 7$. Aliquots of the resulting suspension were incubated for $1 \mathrm{~h}$ with a mixture of Alexa 546-streptavidin and Alexa 633streptavidin (in molar ratios of 0.1, 0.13, 0.2, 0.4, 1, 2.5, 5, $7.5,10,12.5$ and 15) by adding calculated volumes of dye solutions. The suspension was centrifuged (Eppendorf $5415 \mathrm{C})$ at $13,147 \times g$ for $15 \mathrm{~min}$ and washed with PBS. The beads were then immobilized on a Cell-Tak coated cover slip by incubation for $45 \mathrm{~min}$. Excess liquid was removed from the cover slips and dried in air. Fresh PBS buffer was added to the sample before measurement in the confocal microscope.

\section{Microscopy}

Measurements (of Alexa 546-Alexa 633 and CFP-YFP) were carried out in a Zeiss 510 (Zeiss 510 Meta for CFP-YFP) confocal laser scanning microscope with a Plan-Apochromat $63 \times$, NA 1.4 oil objective lens. Green fluorescent protein (GFP)-red fluorescent protein (RFP) pairs were measured in a Leica SP2 AOBS microscope with a Plan-Apochromat $40 \times$, NA 1.25 oil objective. Excitation light intensities were calibrated in front of the objective with a laser power-metre (PD300 and Nova II, Ophir or Biospherical Instruments OSL). From the measurements of power levels, the excitation rate constants $k_{d}^{+}, k_{a}^{+}\left(\lambda_{D, \mathrm{ex}}\right)$ and $k_{a}^{+}\left(\lambda_{A, \mathrm{ex}}\right)$ were estimated with the help of calculated point-spread-functions. For bead and cell measurements the mean power inside one Airy disc was considered a suitable estimate. From the estimated power density and the absorption cross section for the different dyes excitation rate constants were calculated.
CFP-YFP measurements

CHO cells expressing CFP and YFP were excited with a $405 \mathrm{~nm}$ blue laser diode [resulting in $k_{d}^{+}=0.01 \mathrm{~ns}^{-1}$, irradiance $=67 \mathrm{~kW} / \mathrm{cm}^{2} ; k_{a}^{+}\left(\lambda_{D, \mathrm{ex}}\right)$ could be neglected in this case] and the $514 \mathrm{~nm}$ line of an argon ion laser (beamsplitter in front of the lasers, Zeiss HFT 405/514). The common beamsplitter for both detectors was NFT 490. The detectors were equipped with the bandpass filters Zeiss BP 420-480 (channel 1, CFP) and Zeiss BP 530-600 (channel 2, YFP). The pinholes were set to 1 Airy unit. The pixel-dwell-time was $1.3 \mu$ s. For photobleaching in some of the experiments, the sample was irradiated at $514 \mathrm{~nm}$ for $60 \mathrm{~s}$ on a $50 \times 50$ pixel square area.

For fast saturation measurements, three frames (both channels 1 and 2) differing in excitation protocols were acquired in succession: first frame, linear (low level) donor excitation, acceptor light off; second frame, linear acceptor excitation, donor light off; third frame, linear donor excitation and strong acceptor excitation to induce frustration of the acceptor. The crosstalk of YFP (due to potential filter leakage) into channel 1 under saturating light emission was negligible based on the measurements of cells transfected with an empty YFP vector (YFP only). To correct for acceptor photobleaching due to saturating acceptor excitation light during the acquisition of a single image, we estimated the amount of bleaching by the measured acceptor intensity $\left(I_{A \text {,pre }}\right)$ excited with linear acceptor excitation before the saturation light and $I_{A \text {,post }}$ measured after the saturation light obtained from the beginning of the next series of three measurements. We assumed that the measured signal during saturation can be described as the mean of hypothetical saturated signals before and after bleaching, the ratio of which correspond to the one determined above. The unknown saturated acceptor intensity before bleaching is then given by $I_{A \text {, sat,pre }}=2 I_{A \text {, sat }} /(1+$ $I_{A \text {,post }} \Lambda_{A \text {,pre }}$ ). Likewise the donor signal was corrected for acceptor photobleaching (resulting in additional donor dequenching during acceptor saturation) using $I_{D \text {, sat,pre }}=2$ $I_{D \text {,sat }} /\left(1+I_{D \text {,post }} / I_{D \text {,pre }}\right)$, employing the donor intensity $\left(I_{D \text {,pre }}\right)$ measured before the saturation light and $I_{D \text {,post }}$ measured after the saturation light.

\section{Alexa 546-Alexa 633 bead measurements}

The coated beads were excited at a wavelength of $543 \mathrm{~nm}$ (Helium Neon laser; donor excitation light resulting in $k_{d}^{+}=0.0014 \mathrm{~ns}^{-1}$ (this corresponds to an irradiance at the sample of $\left.3 \mathrm{~kW} \mathrm{~cm}{ }^{-2}\right)$ and a crosstalk of $k_{a}^{+}\left(\lambda_{D, \mathrm{ex}}\right)=$ $0.0003 \mathrm{~ns}^{-1}$ and $633 \mathrm{~nm}$ (Helium Neon laser; acceptor excitation light) of the confocal microscope. The beamsplitter separating the detectors from both lasers was Zeiss HFT UV/488/543/633. The individual detectors were 
equipped with the bandpass filters Zeiss BP560-615 (Alexa 546) or Zeiss LP 560-615 (Alexa 633). The confocal pinholes were closed to 1 Airy unit. Images were recorded with a pixel-dwell-time of $1.6 \mu$ s. For the excitation intensity series of measurements, the laser intensities were adjusted with the built-in acousto-optical tuneable filter of the microscope. Data were recorded using given donor $(546 \mathrm{~nm})$ and acceptor $(633 \mathrm{~nm})$ excitation intensities. We used an analogue measuring protocol as in CFP-YFP measurements. Three frames differing in excitation protocols were acquired in succession: first frame, linear (low level) donor excitation, acceptor light off; second frame, linear acceptor excitation, donor light off; third frame, linear donor excitation and strong acceptor excitation. Photobleaching correction was also done as in CFP-YFP measurements. For each intensity measurement, five different beads were recorded. For photobleaching in some of the experiments, the sample was irradiated at $633 \mathrm{~nm}$ for $60 \mathrm{~s}$ on a full area of the bead. For saturation efficiency measurements in Fig. 3d, a saturation light of $k_{a}^{+}=$ $0.06 \mathrm{~ns}^{-1}$ was used. In this intensity range, photobleaching of the acceptor by saturation light could be neglected in the efficiency estimation.

\section{RFP-GFP measurements}

To measure T cells expressing GFP and monomeric RFP1 in the Leica TCS-AOBS microscope, excitation was with the $488 \mathrm{~nm}$ line of an argon ion laser [resulting in $k_{d}^{+}=$ $0.024 \mathrm{~ns}^{-1}$, irradiance $=43 \mathrm{~kW} / \mathrm{cm}^{2} ; \quad k_{a}^{+}\left(\lambda_{D, \mathrm{ex}}\right)$ was neglected] and with $561 \mathrm{~nm}$ light of a solid state laser [resulting in $k_{a}^{+}\left(\lambda_{D, \text { ex }}\right)=0.23 \mathrm{~ns}^{-1}$,irradiance $=720 \mathrm{~kW} /$ $\mathrm{cm}^{2}$ for saturating illumination]. The fluorescence emission was detected with a PMT in the spectral range of 500-530 nm (channel 1, GFP) and 570-700 nm (channel 2, RFP). Pinholes were set to 1 Airy unit. The scanning frequency was $1,000 \mathrm{~Hz}$. For photobleaching in some of the experiments, the sample was irradiated at $561 \mathrm{~nm}$ for $60 \mathrm{~s}$.

For fast saturation measurements, four frames (both channels 1 and 2) differing in excitation protocols were acquired in succession: first frame, linear (low level) donor excitation, acceptor light off; second frame, linear acceptor excitation, donor light off; third frame, linear donor excitation and strong acceptor excitation to induce frustration of the acceptor; forth frame repetition of the first frame (the average from frame 1 and 4 was used in the image analysis). The crosstalk of RFP (due to potential filter leakage) into channel 1 under saturating light emission was negligible based on measurements of cells transfected with an empty RFP vector (RFP only). Also spectral emission unmixing (see below) was not necessary as the emission of GFP and RFP into the respective channels was not detectable in this microscope. Multiphoton fluorescence lifetime imaging (FLIM) measurements were performed according to (Peter et al. 2005).

\section{Spectral emission unmixing}

The spectra of Alexa 546 and Alexa 633 obtained from beads with pure dye coatings were used as fingerprints for the spectral unmixing matrices (Beutler et al. 2002; Gu et al. 2004). The spectra for Alexa 633 were determined under highest acceptor excitation intensity of Fig. $3 \mathrm{~b}$. Individual CFP and YFP spectra were obtained from Jurkat $\mathrm{T}$ cells (a human $\mathrm{T}$ leukaemia cell line) transfected with CFP and YFP alone. The unmixed images $A$ of donor and acceptor were obtained from

$A=N^{-1} f$

where $N=\left(\begin{array}{ll}\bar{I}_{\text {Dch1 }} & \bar{I}_{\text {Ach1 }} \\ \bar{I}_{\text {Dch2 }} & \bar{I}_{\text {Ach2 }}\end{array}\right), \bar{I}_{\text {Dch1 }}, \bar{I}_{\text {Dch2 }}, \bar{I}_{\text {Ach1 }}, \bar{I}_{\text {Ach2 }}$ : pure donor or acceptor signals (mean of respective image in a common region of interest) on channel 1 and $2 A=\left(\begin{array}{c}D_{k} \\ A_{k}\end{array}\right)$,

$D_{k}, A_{k}$ : unmixed donor and acceptor images, and $f=$ $\left(\begin{array}{c}I_{\mathrm{ch} 1} \\ I_{\mathrm{ch} 2}\end{array}\right), I_{\mathrm{ch} 1}, I_{\mathrm{ch} 2}$ : measured images on channels 1 and 2.

For over-determined systems $N^{-1}$ should be replaced by $\left(N^{\mathrm{T}} N\right)^{-1} N^{\mathrm{T}}$ to obtain a least squares solution.

Sensitised emission measurements

On Alexa beads with different stoichiometric ratios were carried out by determining the FRET efficiency (Berney and Danuser 2003) according to

$E_{\mathrm{sensi}}=1-\frac{c_{\mathrm{Dal}} D_{\mathrm{ac}}}{c_{\mathrm{DAc}} D_{\mathrm{al}}}$

where $c_{\text {Dal }}=$ donor concentration on bead with donor coating only; $c_{\mathrm{DAc}}=$ donor concentration on bead with donor and acceptor coating; $D_{\mathrm{ac}}=$ unmixed donor intensity from bead with donor and acceptor coating; $D_{\mathrm{al}}=$ unmixed donor intensity from bead with donor coating only.

satFRET estimation

FRET efficiencies based on excited state saturation of the acceptor were estimated according to (Jares-Erijman and Jovin 2003):

$E_{\text {sat }}=1-\frac{\alpha_{\text {sat }}}{I_{\text {DonorSat }} / I_{\text {Donor }}+\alpha_{\text {sat }}-1}$

where $\alpha_{\text {sat }}=$ degree of acceptor saturation obtained from its calibration curves for the specific dyes; $I_{\text {Donor }}=$ spectrally unmixed donor intensity when in the ground state; 
$I_{\text {DonorSat }}=$ spectrally unmixed donor intensity with the acceptor saturated.

Equation (5) is obtained by assuming a fraction $\left(\alpha_{\text {sat }}\right)$ of molecules incapable of FRET due to acceptor saturation. The measured intensity can thus be written as:

$I_{\text {DonorSat }}=\left(1-\alpha_{\text {sat }}\right) I_{\text {Donor }}+\alpha_{\text {sat }} I_{\text {noFRET }}$

with $I_{\text {noFRET }}$ being the intensity obtained in the absence of FRET. Together with the conventional donor intensity based FRET relationship [see the analogous Eq. (13) below for the case of photobleaching], one obtains Eq. (5) by eliminating $I_{\text {noFRET }}$.

For the derivation of Eq. (5) it was further assumed that the donor excitation remains well within the linear regime, and it was assumed that the degree of acceptor saturation does not depend on whether the donor is in its excited or ground state. Detailed simulations have revealed that such an assumption is not entirely justified if the donor has a lifetime which is considerably longer than that of the acceptor (e.g. in the case of quantum dots donors). In this case a more detailed treatment is necessary, which will be published elsewhere. In our case the maximal error introduced using Eq. (5) (instead of the more detailed model) was $10 \%$ (for $\left.\alpha_{\text {sat }}=0.54\right)$.

When calculating any non-linear functional dependence (such as $E_{\text {sat }}$ ) from ROIs of pixelized data one has to ensure that the summation or averaging operations are always performed prior to the application of the corresponding non-linear equation. This avoids an unwanted bias that can be introduced by the interplay of the non-linear equation and the statistical variability of the raw data. In our analysis this "rule of first averaging the ROIs" was implemented in the analysis.

Effect of photobleaching of acceptor molecules on the FRET efficiency estimation by Eq. (5) in FRET pairs of biosensors like CFP-YFP or GFP-RFP

The estimated efficiency measured by the saturation technique of a population of FRET pairs with a share of acceptors photobleached is given by $E_{\text {sat }}^{\prime}$ analogue to Eq. (5):

$E_{\text {sat }}^{\prime}=1-\frac{\alpha_{\text {sat }}}{I_{\text {DonorSat }}^{\prime} / I_{\text {Donor }}^{\prime}+\alpha_{\text {sat }}-1}$

where $\alpha_{\text {sat }}=$ degree of acceptor saturation obtained from its calibration curves for the specific dyes; $I^{\prime}$ Donor $=$ spectrally unmixed donor intensity with all acceptors in the ground state, measured when a fraction of acceptors has been photobleached [defined in Eq. (7); and $I_{\text {DonorSat }}^{\prime}=$ spectrally unmixed donor intensity with the acceptor saturated (fraction $\alpha_{\text {sat }}$ ) measured when a fraction of acceptors has been photobleached (defined in Eq. (7)]. The measured intensity can be separated into two contributions, with the two extreme cases given by the situation in which all acceptors are intact (measurements $I_{\text {Donor }}$ and $I_{\text {DonorSat }}$ ) or destroyed (measurements $I_{\text {Donor,Bleached, now independent of saturation level). The }}$ measurement under partial bleaching conditions is thus linearly dependent on the relative amount of intact acceptor molecules $(\beta)$ :

$I_{\text {Donor }}^{\prime}=\beta I_{\text {Donor }}-(1-\beta) I_{\text {Donor,Bleached }}$

$I_{\text {DonorSat }}^{\prime}=\beta I_{\text {DonorSat }}-(1-\beta) I_{\text {Donor,Bleached }}$

Equation (5), which is valid for the non-photobleached situation, can be solved for the measured donor emission under acceptor saturation conditions:

$I_{\text {DonorSat }}=\frac{I_{\text {DonorSat }}\left(1-E_{\mathrm{sat}}+\alpha_{\mathrm{sat}} E_{\mathrm{sat}}\right)}{1-E_{\mathrm{sat}}}$

The condition of all acceptor molecules bleached $[\beta=0$ in Eq. (7)] would be equivalent to the non-bleached fully saturated situation, thus setting $\alpha_{\text {sat }}$ to unity in Eq. (5) and replacing $I_{\text {DonorSat }}$ with $I_{\text {Donor,Bleached }}$ whilst keeping the non-bleached linear donor emission. This condition yields $I_{\text {Donor,Bleached }}=\frac{I_{\text {Donor }}}{1-E_{\text {sat }}}$ with $E_{\text {sat }}$ : the efficiency estimated by Eq. (5) where all acceptors are intact. Taking Eqs. (6)-(8) and these considerations together one obtains the following simple result:

$E_{\mathrm{sat}}=\frac{E_{\mathrm{sat}}^{\prime}}{\beta}$

Correction of $\mathrm{E}_{\text {sat }}^{\prime}$ for acceptor photobleaching in time series

The calculation of the corrected FRET efficiency from time series data would be straightforward with Eq. (9), if the extent of acceptor bleaching were known for every frame. However, with living cells problems arise in the estimation of $\beta$ with data solely based on the acceptor emission intensity due to time-dependent changes of cell morphology and focus drift. Therefore, we used the change in the ratio of donor and acceptor concentrations for the photobleaching estimation. A FRET independent (unquenched) donor emission had first to be estimated in every frame $n$. This was achieved using Eq. (6) with $\alpha_{\text {sat }}$ set to unity and solving for the corresponding donor emission under complete acceptor saturation.

$c_{\text {Donor }}(n) \sim I_{\text {DonorUnquenched }}(n)=\frac{I_{\text {Donor }}^{\prime}(n)}{1-E_{\text {sat }}^{\prime}(n)}$

The acceptor concentration can directly be estimated from the measurement obtained under low intensity acceptor excitation conditions (no donor excitation): 
$c_{\text {Acceptor }}(n) \sim I_{\text {Acceptor }}(n)$

It is not recommendable to use high intensity measurements since such data would have a different point-spread-function due to the saturation and a higher contribution from other (out-of-focus) signals. The fraction of remaining acceptor molecules $\beta(n)$ at each time point in the series can be estimated from:

$\beta(n)=\frac{I_{\text {Acceptor }}(n) / I_{\text {DonorUnquenched }}(n)}{I_{\text {Acceptor }}(1) / I_{\text {DonorUnquenched }}(1)}$

where the frame number $n=1$ indicates the first measured frame. Equation (12) can be used in combination with Eq. (8) to calculate $E_{\text {sat }}$ for every time point of the time series. This estimation of acceptor bleaching based on relative changes is robust to changes in the total concentration of biosensor in the image plane over time.

FRET efficiencies by acceptor photobleaching were estimated according to Eq. (13) (Bastiaens and Jovin 1998)

$E_{\text {bleach }}=1-\frac{I_{\text {Don,before }}}{I_{\text {Don,after }}}$

with $I_{\text {Don,before }}=$ spectrally unmixed donor intensity when all acceptors are intact prior to photobleaching; and $I_{\text {Don,after }}=$ spectrally unmixed total donor intensity after acceptors are completely photobleached (corresponding to $I_{\text {noFRET }}$ above).

\section{CHO (Chinese hamster ovary) cell preparation}

$\mathrm{CHO}$ cells were transfected with a plasmid encoding for a protein kinase $\mathrm{C}$ reporter (CFP-YFP-tagged, CKAR construct) which exhibits FRET constitutively in untreated cells (Violin et al. 2003).

$\mathrm{T}$ cell culture and transient transfection

Jurkat cell line-Human leukaemic $\mathrm{T}$ cells were cultured in RPMI1640 medium (life Technologies Ltd., UK) supplemented with 10\% FCS (Sigma-Aldrich Co), 1\% glutamine, $100 \mu / \mathrm{ml}$ penicillin and $10 \mu \mathrm{g} / \mathrm{ml}$ streptomycin (Life Technologies), at $37^{\circ} \mathrm{C}$ in a humidified $5 \% \mathrm{CO}_{2}$ incubator.

Electroporation: Jurkat T cells $\left(10^{7}\right)$ were electroporated in $250 \mu \mathrm{l}$ serum free RPMI medium (phenol red free, 25 $\mathrm{mM}$ Hepes) containing $40 \mu \mathrm{g}$ of plasmid DNA at $260 \mathrm{~V} /$ $950 \mu \mathrm{F}$ using the Gene Pulser II electroporation system (Biorad, US). The cells were allowed to recover for $24 \mathrm{~h}$ before plating on antibody-coated cover slip.

CFP-Raichu-YFP probes for reporting localised small RhoGTPase activities (Cdc42, Rac1, RhoA) in cells have been reported previously (Itoh et al. 2002) and were obtained from Professor M. Matsuda (Osaka University, Japan). To make the GFP-Raichu-RFP version, the section between the YFP and CFP fluorophores was excised and inserted between the RFP and EGFP [in the pEGFP-N1 vector (Clontech, US) that has been modified by the addition of mRFP1 and altering the multiple cloning sites]. The KRasCAAX sequence was then cloned after the EGFP, with the resulting construct maintaining the same linkers as the original.

Ligand-dependent activation assay for Jurkat $\mathrm{T}$ cells

For live imaging, chambered cover glasses (LabTek) were coated overnight with goat anti-mouse (Fc specific) $\mathrm{F}(\mathrm{ab}$ ')2 fragment $(10 \mu \mathrm{g} / \mathrm{ml})\left(\right.$ Sigma) at $4^{\circ} \mathrm{C}$. The chamber slides were then incubated with the stimulatory anti-CD3 (UCHT1) and anti- $\beta 1$ integrin (12G10) monoclonal antibodies, at a final concentration of $10 \mu \mathrm{g} / \mathrm{ml}$ in PBS, for $2 \mathrm{~h}$ at $37^{\circ} \mathrm{C}$. Excess antibody was removed. Jurkat cells were washed and resuspended in RPMI without phenol red, containing $10 \%$ FCS and $25 \mathrm{mM}$ Hepes, then plated at a density $2 \times 10^{6}$ cells $/ \mathrm{ml}$ minutes before being imaged.

For the preparation of fixed samples the same procedures were used for the transfection and antibody coating. The cells were allowed to attach on antibody-coated cover slips for 5,10 , and $30 \mathrm{~min}$ at $37^{\circ} \mathrm{C}$ in a humidified $5 \% \mathrm{CO}_{2}$ incubator. Cells were then fixed with $4 \%(w / v)$ paraformaldehyde, permeabilized with $0.2 \%$ Triton X-100 for 5 min and then quenched in $1 \mathrm{mg} / \mathrm{ml}$ sodium borohydride for $10 \mathrm{~min}$. The cover slips were then mounted onto slides with immuno-fluore mounting medium.

\section{Results}

To test the theoretical basis for satFRET, the steady-state solution of the differential equation system shown in Fig. 1 [given in Eq. (1A) for the acceptor- and Eq. (2A) for the donor-S-state populations] was plotted at a fixed donor excitation rate $\left(k_{d}^{+}=0.002 \mathrm{~ns}^{-1}\right)$ for a range of acceptor excitation rates, and seven different transfer efficiencies in Fig. 2. Panel A shows the excited donor $\left(D^{*}\right)$ fraction proportional to the donor fluorescence intensity. The state $D^{*}$ is populated by direct donor excitation [the fluorescence intensity is proportional to the excited singlet state population according to Eq. (2B)]. With increasing excitation of the acceptors and occupation of the transferincompetent state, the donors become increasingly dequenched in FRET pairs with efficiencies, i.e. the $D^{*}$ fractional population increases. In panel B of Fig. 2 the $A^{*}$ fraction proportional to the acceptor fluorescence intensity can be seen. The initial $A^{*}$ population is finite even in the absence of direct acceptor excitation due to energy transfer from the directly excited donors. With increasing acceptor excitation, the acceptor fluorescence increases linearly and 
(A)
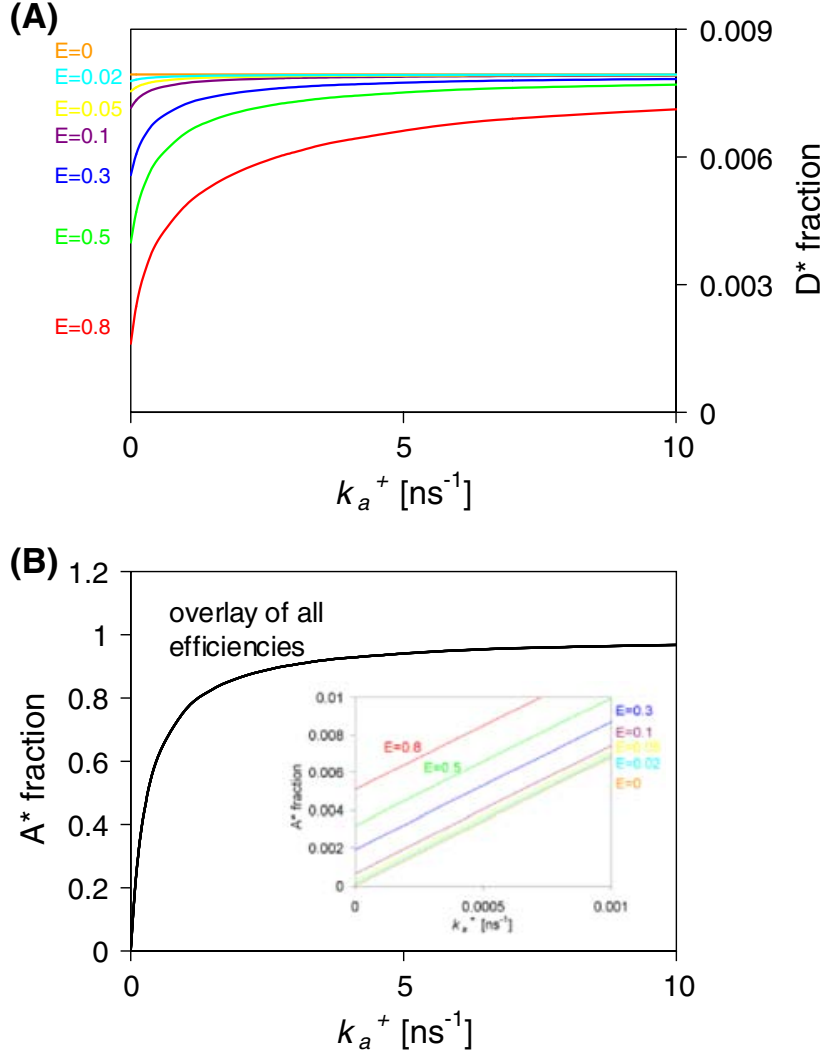

Fig. 2 Simulated donor and acceptor singlet states in FRET pairs of Alexa 546 (donor) and Alexa 633 (acceptor) according to Eqs. (1A) and (2A). The donor is excited in the linear regime $\left(k_{d}^{+}=0.002 \mathrm{~ns}^{-1}\right)$. The acceptor excitation intensity $\left(k_{a}^{+}\right)$was varied. The fluorescence lifetimes ( $\tau$ 's) of Alexa 546 and Alexa 633 were assumed to be 4 and $3.2 \mathrm{~ns}$, respectively (Molecular 2004), corresponding to $k_{d}^{-}=(1 / 4) \mathrm{ns}^{-1}$ and $k_{a}^{-}=(1 / 3.2) \mathrm{ns}^{-1}$. Different FRET pairs with transfer efficiencies of $E=0,0.02,0.05,0.1,0.3,0.5$ and 0.8 were considered. a $D^{*}$ (excited donor) fraction, b $A^{*}$ (excited acceptor) fraction, inlet of $\mathbf{b}$ same as $\mathbf{b}$ but range of $k_{a}^{+}$was $0-0.001 \mathrm{~ns}^{-1}$

then approaches a maximal plateau value. In Fig. $2 b$ no difference can be seen between the curves for different efficiencies $E$. In the inset of Fig. $2 b$ it becomes more visible that the initial offset is higher for higher efficiencies $E$ and zero for $E=0$. The donors corresponding to the uncoupled $(E=0)$ FRET pairs in Fig. 2a are populated to a constant higher level than those with $E \neq 0$, i.e. independently of the degree of acceptor saturation.

The next step was to test the prediction of Fig. 2 with an actual FRET system in practice. Beads were loaded with different molecular ratios of Alexa 546 and 633. These beads can be regarded as multiple donor-acceptor pair systems varying in the ratio of donors and acceptors. The integrated FRET efficiencies (Berney and Danuser 2003) of the particles can be changed by shifting the donor acceptor stoichiometry. The results of these measurements are displayed in Fig. 3.
Figure 3a shows the dependence of spectrally unmixed detected fluorescence of Alexa 546 alone on the additional intensity applied at the acceptor excitation wavelength. As expected, the donor fluorescence exhibited no measurable increase. Particles carrying only Alexa 633 (acceptor, Fig. 3b) were measured in the same manner. With donor but no acceptor $(633 \mathrm{~nm})$ excitation light present, the acceptor exhibited fluorescence due to its direct excitation at the donor excitation wavelength $(543 \mathrm{~nm})$. With increasing acceptor excitation light intensities, the acceptor was driven into saturation. The donor fluorescence fluctuated about zero, as expected from spectral unmixing in the absence of donor.

The saturation behaviour of FRET particles with donor:acceptor molar ratio of 0.2 is featured in Fig. 3c. The initial acceptor fluorescence was finite in the absence of acceptor excitation light due to direct donor excitation and FRET transfer. At higher acceptor excitation intensities, the acceptor signal became saturated. By fitting Eq. (1A) and (1B) to the acceptor curve, the degree of saturation at the highest excitation intensity of the acceptor could be estimated $\left(\alpha_{\text {sat }}=0.33 \pm 0.01\right)$. [In general, fitting of Eq. (1A) and (1B) is required to estimate the degree of acceptor saturation, using at least three experimental data points and determination of $k_{f, a}$. If $k_{\mathrm{a}}^{-}$is unknown or if it changes from experiment to experiment it can be fitted as well. But more experimental data points are then required.

The donor fluorescence was clearly dequenched as the acceptor became increasingly saturated.

In the same manner, saturation based FRET efficiencies were estimated using Eq. (5) for particles with different donor and acceptor stoichiometries. Those were compared to the efficiencies measured by sensitised emission [Eq. (4)] and acceptor photobleaching [Eq. (13)]. A clear correlation between the two methods can be seen in Fig. 3d.

The frustrated FRET method was tested with $\mathrm{CHO}$ cells containing a CFP-YFP construct in the closed state. We anticipated a homogenous FRET efficiency throughout each cell. In an initial experiment the behaviour of the donor and acceptor fluorescence was tested for different acceptor excitation intensities at $514 \mathrm{~nm}$ and the excitation intensity at $405 \mathrm{~nm}$ (donor excitation) constant (Fig. 4).

The results agreed well with the bead measurements in Fig. 3c. The acceptor fluorescence showed a linear increase at low acceptor excitation intensities and approached a maximal value. The donor fluorescence increased as the acceptor reached saturation. Fitting Eq. (1B) to the data yielded an estimate of the acceptor saturation achieved for a given acceptor excitation intensity. At the highest acceptor excitation intensity the estimated $\alpha_{\text {sat }}$ was $0.31 \pm$ 0.02 . Nevertheless, although the average $E_{\mathrm{sat}}$ estimation for all points except the two highest excitation intensities was 
Fig. 3 Fluorescence signals of spectrally unmixed donor and acceptor images from bead measurements. Images were recorded with linear donor excitation $(543 \mathrm{~nm})$ and different acceptor excitation rates [irradiance $=\left(k_{a}^{+}\right)\left(\lambda_{A, \mathrm{ex}}=\right.$ $633 \mathrm{~nm}) 8.1 \times 10^{-7} \mathrm{~ns} \mathrm{kW/}$ $\mathrm{cm}^{2}$ ]. a Donor (Alexa 546) alone, b acceptor (Alexa 633) alone, $\mathbf{c}$ donor in the presence of acceptor at a donor:acceptor ratio of $0.2, \mathbf{d}$ comparison of FRET efficiencies estimated by sensitised emission and acceptor photobleaching with those estimated by excited state saturation. The measurements of five beads were averaged per data point. Error bars represent the standard deviation between measurements
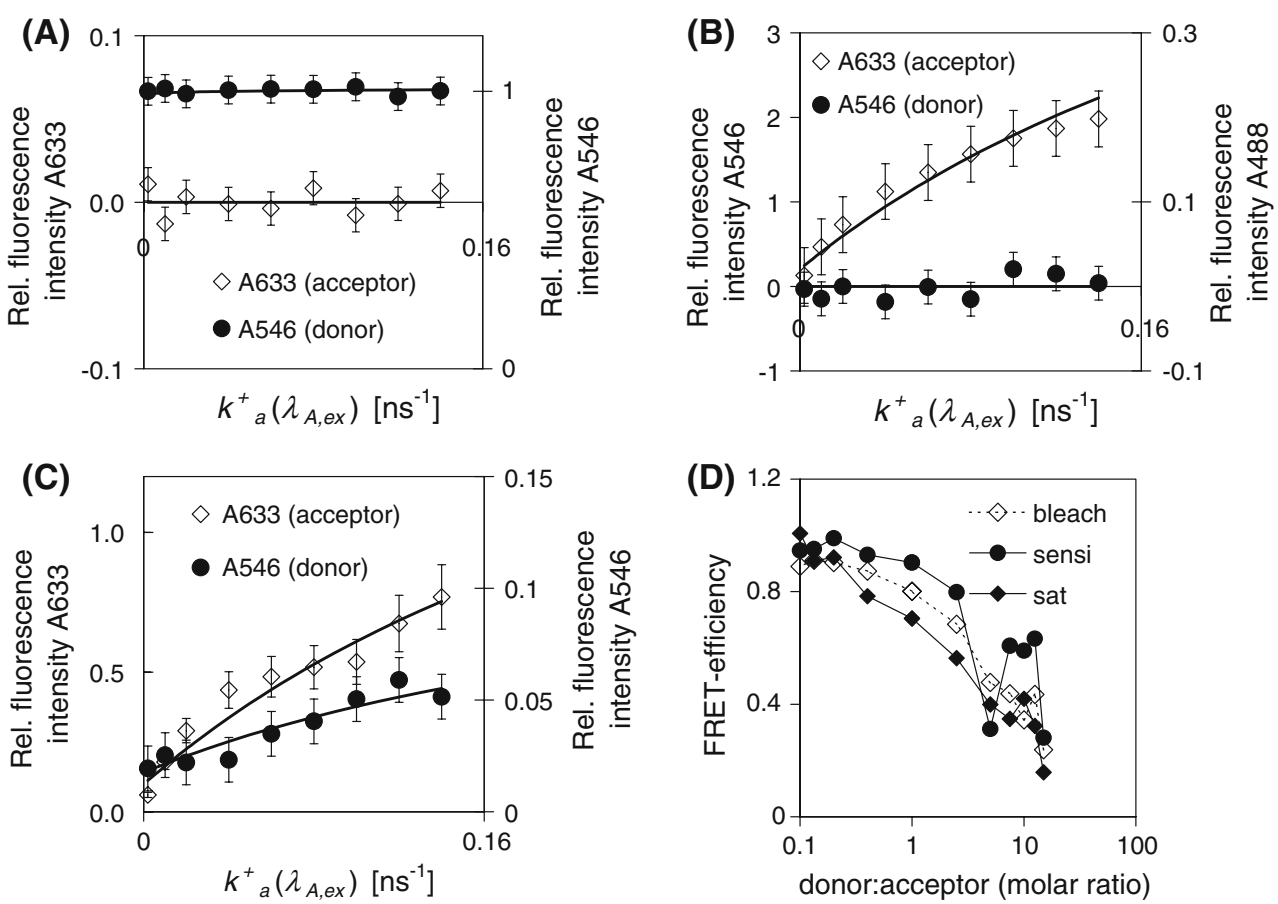

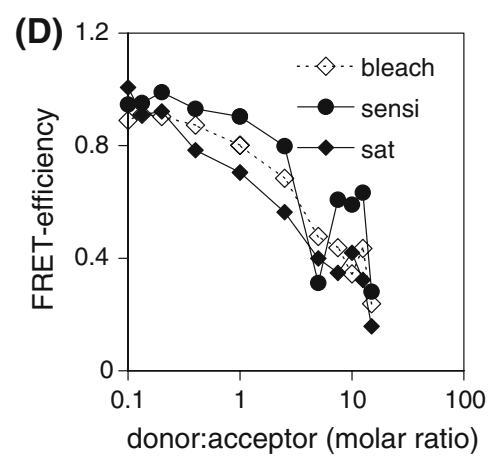

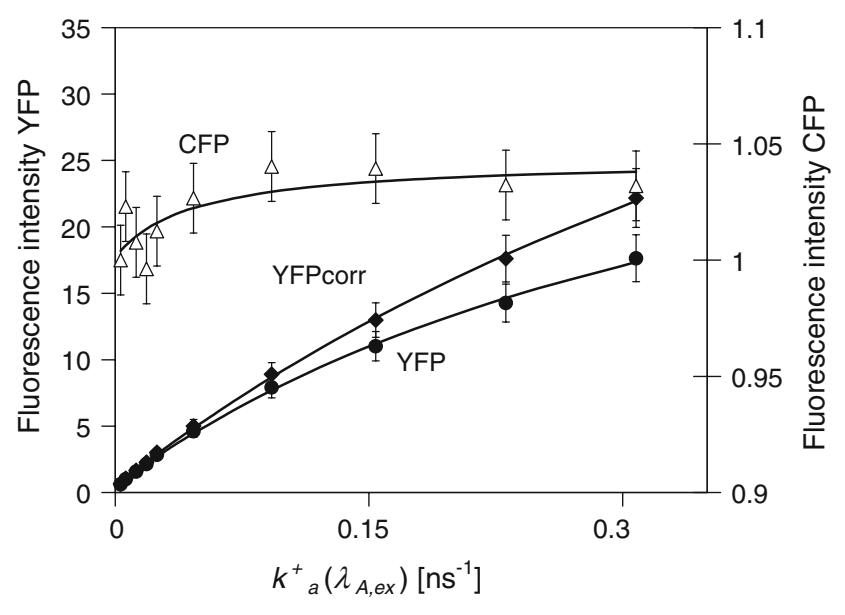

Fig. 4 Fluorescence of $\mathrm{CHO}$ cells under different acceptor excitation rates [irradiance $=\left(k_{a}^{+}\right)\left(\lambda_{A, \mathrm{ex}}=514 \mathrm{~nm}\right) 1.2 \times 10^{-6} \mathrm{~ns} \mathrm{~kW} / \mathrm{cm}^{2}$ ]. (open circle, CFP donor; filled diamond, YFP acceptor; filled circle, YFP acceptor corrected photobleaching see "Methods"). The donor excitation intensity $(405 \mathrm{~nm})$ was in the linear range. The solid lines represent a fit to the data points according to Eqs. (1B) and (2B). The lifetime of CFP was assumed to be $2.3 \mathrm{~ns}$ (Biskup et al. 2004)

0.25 (expected was 0.3 see also Fig. 5), the estimation of $E_{\text {sat }}$ for the last two points (0.1) was too low and the photobleaching algorithm (described in the "Methods" under CFP-YFP measurements) could not compensate for photobleaching at these acceptor excitation intensities. Thus, we chose to work at lower excitation intensity in subsequent experiments.

To check the applicability in a time series on a $\mathrm{CHO}$ cell seven measurements were carried out employing the saturation based measuring protocol (Fig. 5). A square region of the YFP acceptor was photobleached after the second measurement. Saturation efficiencies were calculated before and after the photobleaching event.

In Fig. 5a and c the donor and acceptor images of the non-photobleached cell can be seen. After photobleaching, the acceptor fluorescence decreased in the selected region (Fig. 5c, d), while the donor signal increased accordingly (Fig. 5b). The distribution of saturation-based (Fig. 5e) FRET efficiencies was homogenous as in the case of the values obtained by the photobleaching approach (Fig. $5 \mathrm{~g}$ ). After the bleaching event the saturation data continued to provide results due to the reversibility of the method (Fig. 5f). In the bleached region a decrease in FRET efficiency was clearly seen whilst FRET events in the surrounding unbleached area could still be measured.

The saturation method was tested with live $\mathrm{T}$ cells spread on activating antibodies against $\mathrm{CD} 3$ and $\beta 1$ integrin [at concentrations optimised previously ( $\mathrm{Ng}$ et al. 1999)] that were immobilised on cover glass (Fig. 6). During this ligand-induced activation, a fraction of the GFP-Raichu-RFP probe converted from the open conformation (low FRET efficiency, i.e. GDP-bound) to the closed state (high FRET efficiency, i.e. GTP-bound). FRET efficiencies $E_{\text {sat }}$ [corrected for acceptor photobleaching according to Eqs. (9)-(12)] were estimated throughout this activation process. In the FRET efficiency maps, an increase over time was observed (Fig. 6a). This trend was also visible in the averaged efficiencies (Fig. 6b). The mean acceptor signal lost about $40 \%$ of the initial intensity and indicated a similar decrease in acceptor concentration due to photobleaching. The acceptor signal increased after 


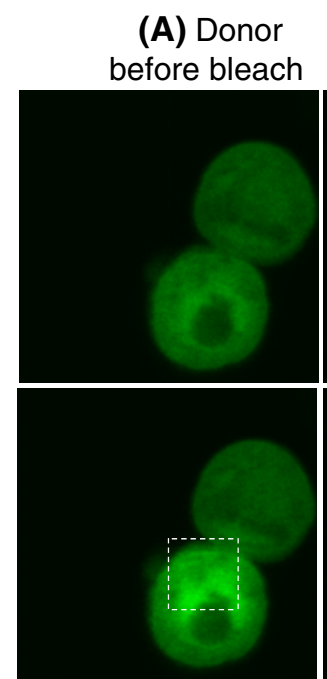

(B) Donor after bleach
(C) Acceptor before bleach
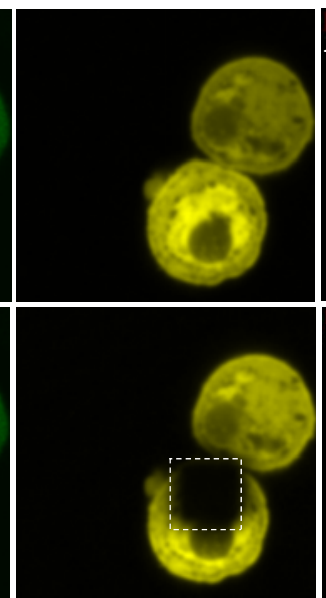

(D) Acceptor after bleach

Fig. 5 CHO cells measured with the frustrated FRET saturation protocol. Acceptor photobleaching was applied after the second measurement. Dashed boxes indicate the bleached region. a donor image when acceptor light was off before photobleaching (low intensity), b donor image when acceptor light was off after photobleaching of indicated square region (low intensity), c acceptor image when acceptor was directly excited prior to photobleaching, $\mathbf{d}$ acceptor image when acceptor was directly excited after photobleaching,

12 min, probably due to diffusion of biosensors from unbleached regions of the cell or movement of the cell. The bleaching corrected $E_{\text {sat }}$ increased over time. Compared to the results of the photobleaching experiments of the fixed cells, $E_{\mathrm{sat}}$ was in good correspondence while $E_{\text {sat }}^{\prime}$ yielded values that were lower than $E_{\text {bleach. }}$ In addition to the experiments shown in Fig. 6, in five other cells exposed to the same treatment as that in Fig. 6a, the acceptor molecules were destroyed completely after $30 \mathrm{~min}$ exposure time and a mean efficiency of $E_{\text {sat }}^{\prime}=-0.02 \pm 0.01$ was determined after photobleaching (data not shown). This result constituted an estimation of the zero point, i.e. in the absence of energy transfer from donor to acceptor.

Figure $6 \mathrm{c}$ shows an assay monitoring the Raichu-Cdc42 activity changes in live $\mathrm{T}$ cells that were in the process of adhering to extracellular substrates (provided here in the form of receptor-specific activating antibodies) on a glass surface. Adhesion does not occur in the absence of a specific activation signal (e.g. anti-mouse $\operatorname{IgG~Fc}$ antibody alone) and most of the receptor-specific activating antibodies we have used stimulate FRET (measured by FLIM) in a time-dependent manner, albeit with variable kinetics making it difficult to provide images of "Unstimulated cells with steady $E$ ". However, a comparison with cells in suspension that were fixed and cytospun onto a cover slip before mounting ("cytospin control" in the attached)

(E) $E_{\text {sat }}$ before bleach

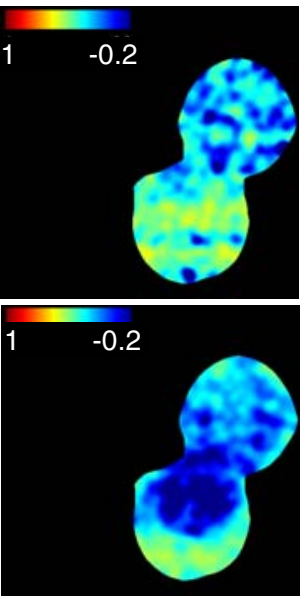

(E) $E_{\text {sat }}$ after bleach
(G) $E_{\text {bleach }}$
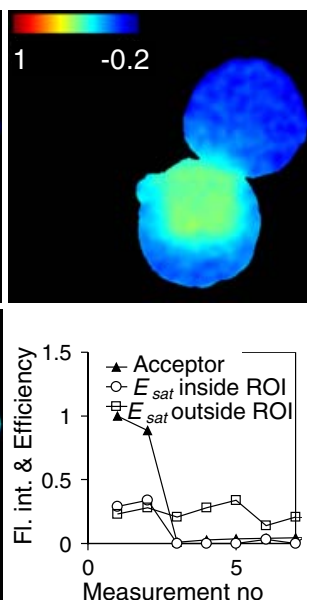

(H) Acceptor \&

$E_{\text {sat }}$ (inside \& outside ROI)

e FRET efficiency based on acceptor excited state saturation using Eq. (5) before photobleaching $\left(\alpha_{\text {sat }}=0.1 \pm 0.005, E_{\text {sat }}=0.32\right)$, f FRET efficiency based on excited state saturation according to Eq. (5) after photobleaching $\left(E_{\text {bleach }}=0.35\right)$, $\mathbf{g}$ FRET efficiency based on acceptor photobleaching calculated by Eq. (13), $\mathbf{h}$ integrated signals of the bleaching and non-bleaching region of the seven measurements showing the acceptor signal with direct excitation and the estimated FRET efficiency based on excited state saturation

provided an appropriate negative control for the Raichu sensor.

\section{Discussion}

The simulation of the differential equation system (Fig. 1) and its time-independent solution in Eqs. (1A) and (2A) demonstrate the feasibility of deactivating acceptor molecules by high acceptor excitation intensity, while the donor is simultaneously excited with a low intensity. This effect can be used to estimate FRET efficiencies according to Eq. (5). Surprisingly, the degree of donor dequenching in Fig. 1a was already evident at a low acceptor excitation intensity. In the simulated example of Alexa 546-Alexa 633 (Fig. 2), low acceptor excitation intensities had a significant effect on the donor dequenching and at $50 \%$ acceptor saturation $85 \%$ of the maximal donor increase was achieved (Fig. 2a, $E=0.8$; for lower transfer efficiencies the increase was even higher). This result demonstrates that relatively low light intensities (in the linear regime) suffices for implementation of the satFRET technique. However, high excitation light intensity is not necessarily a disadvantage, inasmuch as many dyes (e.g. fluorescein) at low concentration photobleaching occurs with a rate constant that is excitation intensity independent and proceeds predominantly via triplet states in the 
Fig. 6 Live T cell measured in an activation assay with the frustrated FRET saturation protocol in time series. Time point zero marks the moment when $T$ cells were added to the antibody-coated cover glass and measurements were started $\left(\alpha_{\text {sat }}\right.$ $=0.62 \pm 0.01)$. $\mathbf{a}$ Donor images and $E_{\mathrm{sat}}$ images, corrected for acceptor photobleaching according to Eqs. (9)-(12), estimated for a single cell inside an intensity based mask (sum of donor and acceptor intensity $>20 \%$ of maximal sum of donor and acceptor intensity). Prior to computing the $E_{\text {sat }}$ images a Gaussian filtering with a $2 \times 2$ kernel was applied. b Acceptor signal, $E_{\text {sat }}^{\prime}$ [uncorrected for acceptor photobleaching, Eq.(6)] and $E_{\text {sat }}$ (corrected for acceptor photobleaching, evaluated for the same cell and mask as in a, error bars show the standard errors). Single data points (closed circles) show the efficiencies and their standard error estimated from acceptor photobleaching experiments of fixed cells with different incubation times on the cover glass. Efficiencies of five fixed cells were averaged in each of the $E_{\text {bleach }}$ estimates. c Multiphoton FLIM measurements of the intramolecular FRET between the GFP and mRFP1 components of the GFPRaichu-Cdc42-mRFP1 biosensor in Jurkat $\mathrm{T}$ cells that have spread on anti-CD3 (UCHT1) + anti-b1 integrin (12G10) 'activating' antibodies for up to $30 \mathrm{~min}$ on cover slips. FRET between GFP and mRFP1 results in shortening of the fluorescent lifetime (tau) of GFP and biexponential decay kinetics are observed

\section{(A)}
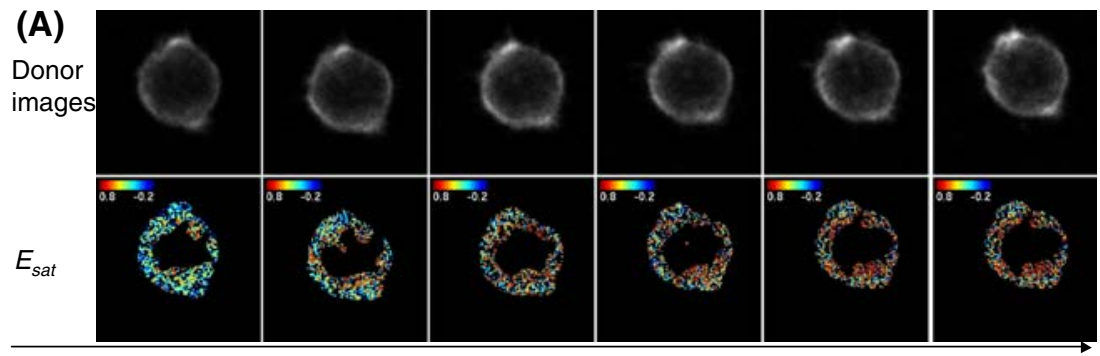

Time [min] 0

6

9

12

15
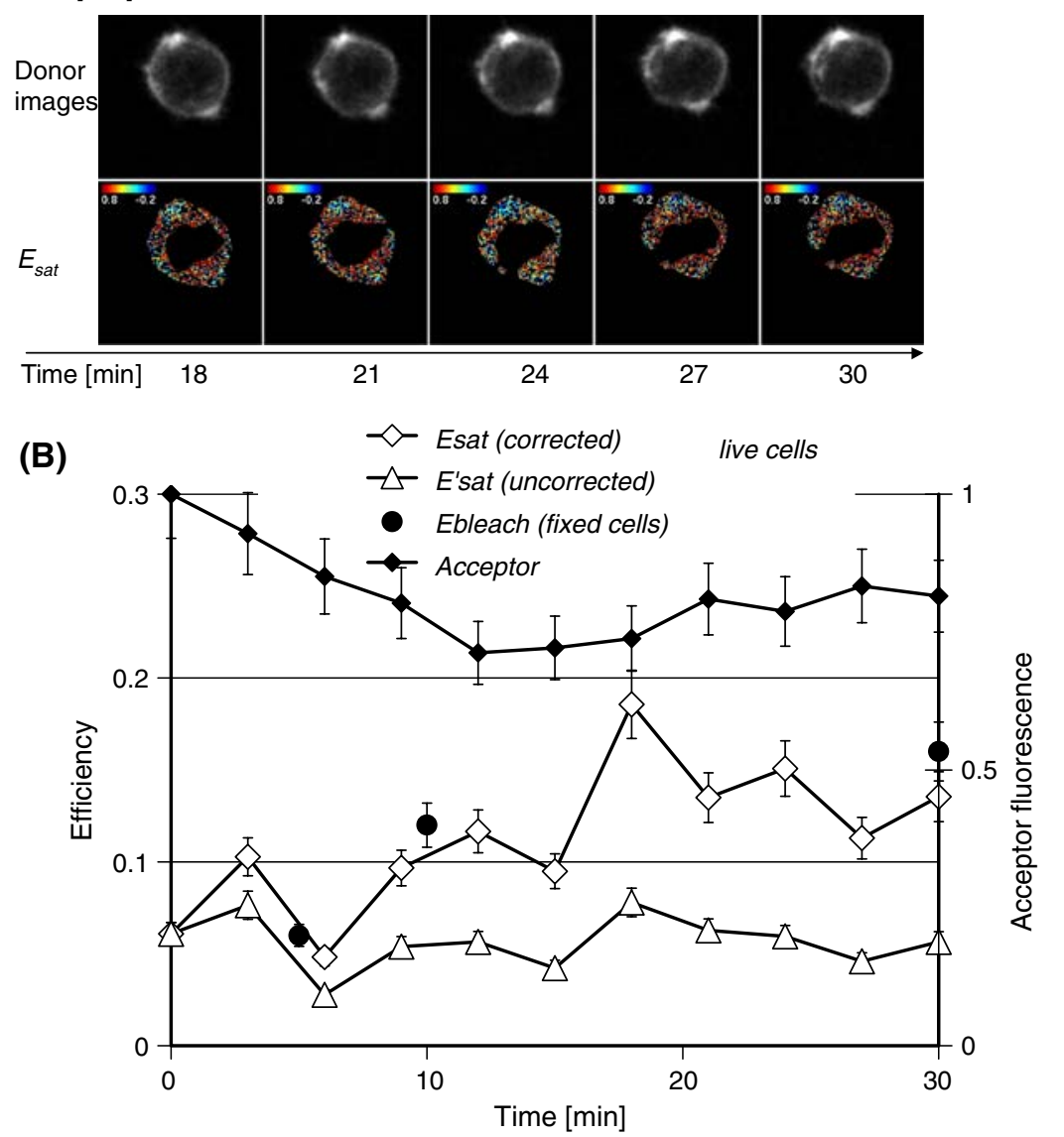

(C)
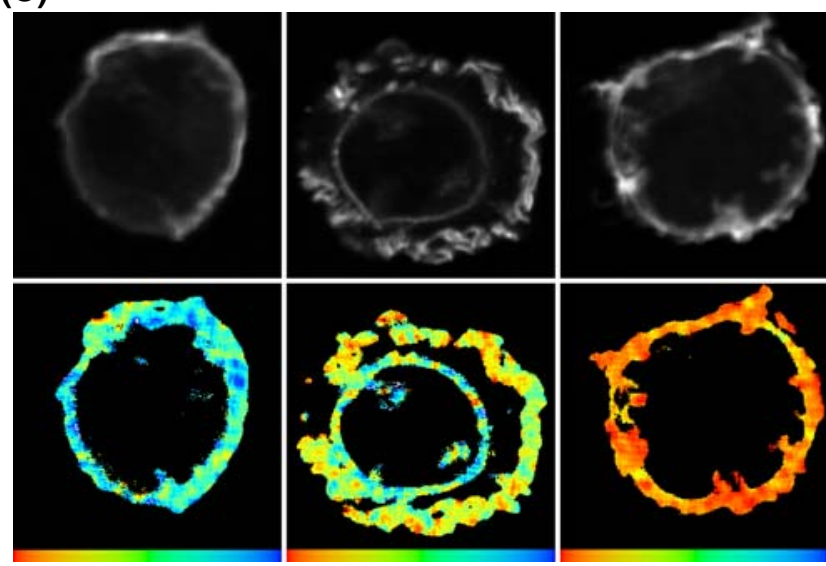

$1.76 \tau(\mathrm{ns})$

$2.061 .76 \tau(\mathrm{ns})$

$2.061 .76 \tau(\mathrm{ns})$

2.06

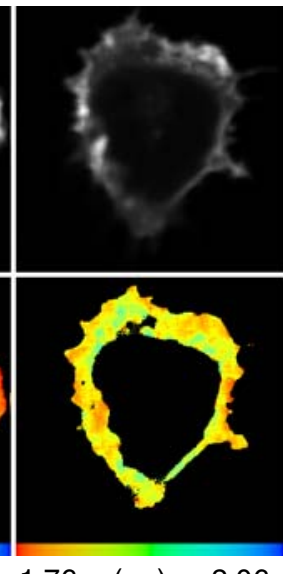

Cytospin

$5 \min$ on $\alpha$ -

$10 \mathrm{~min}$ on $\alpha-$ control CD3/integrin

$30 \mathrm{~min}$ on $\alpha$ CD3/integrin 
presence of oxygen (Song et al. 1995; Song et al. 1996). In contrast, rhodamine-like acceptor molecules have excitation intensity-dependent photobleaching rates (Widengren et al. 1995; Widengren and Rigler 1996) due to the involvement of higher triplet and singlet states. In this case, the use of lower light levels may be desirable. In FRET pairs with a unity donor-to-acceptor-ratio the theoretical description in Eqs. (6)-(11) of the influence of acceptor photobleaching leads to (see "Methods") the simple relation that the estimated efficiencies are proportional to the degree of acceptor photobleaching $\beta$. This allowed for correction of the photobleaching effect even in images in which the total concentration of biosensors changed over time due to diffusion and cellular movement.

The experiments on beads labelled with a single dye showed that the crosstalk (filter breakthrough and additional autofluorescence) could be neglected. Similar results were obtained with the pure YFP and CFP cells and the settings of our particular microscope. The results in Fig. 3 demonstrate that a donor signal increase under acceptor excitation conditions can be readily achieved with light intensities available in commercial confocal microscopes.

The qualitative and quantitative agreement of excited state saturation and acceptor photobleaching (Fig. 3d) was very good, whereas the correspondence between sensitised emission and the two other methods was less so. One can argue that the sensitised emission FRET measurement, calculated according to Eq. (4), can be subject to substantial error, simply because applying mixtures of compounds with different dyes and at high concentration may well lead to problems at high dye density such as masking and quenching. Equation (4) assumes the validity of the concentration ratio (as estimated from the titrating of the dye ligands) and does not account for possible concentration dependent changes in quantum yield. The important and much more interesting feature in Fig. $3 \mathrm{~d}$ is the demonstration that the satFRET response is a robust function of the $D: A$ ratio and shows a decrease with increasing $D: A$ ratio (Berney and Danuser 2003).

A potential concern of the satFRET approach is its underlying assumption that the acceptor excited state is unable to perform FRET. Since also radiation-less FRET can be very effective there may be situations where direct acceptor excitation could even enhance the FRET process. These effects can be incorporated easily into the satFRET formalism. However, they were absent in the systems selected for investigation and reported here.

The usefulness of the satFRET approach was also demonstrated by data obtained from the live cell series using the GFP-Raichu-RFP biosensor. $E_{\text {sat }}$ was in good correspondence when compared to the results of the equivalent photobleaching experiments of fixed cells. For the CFP-YFP couple, the difference between $E_{\text {sat }}$ and
$E_{\text {bleach }}$ could originate from an overestimation of $E_{\text {bleach }}$ due to production of CFP-like fluorophores in the course of photobleaching of YFP (Valentin et al. 2005).

The frustrated FRET method is applicable to biological systems, as shown by Figs. 4 and 5. In the cell experiments of Figs. 4 and 5a, the CFP/YFP pair was selected for the measurements of FRET efficiencies based on excited state saturation after photobleaching (Fig. 5). This choice led to the side effect of acceptor photobleaching at the saturating levels of excitation (Fig. 4) and it was only possible to work with low acceptor saturation intensities in Fig. 5 to reduce unwanted photobleaching in the saturation analysis. The time-resolution of the method and applicability to live cells was clearly demonstrated by the $\mathrm{T}$ cell activation assay in Fig. 6. We found RFP to be more resistant than YFP to photobleaching in the CHO cells. Nonetheless, the degree of acceptor photobleaching was $\beta=0.6$ in the live experiment after eleven measurements at a saturation level of $\alpha_{\text {sat }}=0.62 \pm 0.01$. In other experiments with $\alpha_{\text {sat }}=0.3 \pm$ 0.008 up to 20 measurement were possible (data not shown). The estimated efficiencies could be corrected for acceptor photobleaching by Eqs. (6)-(12) in Fig. 6.

Another way to circumvent the photobleaching problem would be to use other fluorescent proteins with improved photophysical properties (Kremers et al. 2007; Su 2005). Finally, it may be possible to minimise photobleaching by reducing the pixel dwell time and acceptor excitation light intensity.

The pseudocolor FLIM plots (Fig. 6c) for fixed cells expressing a GFP- and mRFP1-tagged Cdc42 Raichu construct allowed us to obtain FRET images of GTPase activity from lifetimes. They show similar kinetics upon comparison with the data obtained from the $E_{\text {sat }}$ calculations (the fluorescence lifetime was shortened after 5, 10 and 30 min compared to the cytospin control).

The inhomogeneity of the excitation beam of the confocal system produced a inhomogenous pattern of acceptor saturation. In case of particles (Fig. 3) and in the other example of the $\mathrm{CHO}$ cells (Figs. 4, 5) the fluorescence emission caused by the non-saturating higher orders of the excitation beam and the flanks of the main beam could be eliminated by closing (to 1 Airy unit) the pinholes in front of the photomultipliers.

In conclusion, significant donor dequenching already takes place in the linear excitation intensity regime (Fig. 2 for the example of Alexa 546-633) and can lead in this region to a donor fluorescence increase of $30 \%$ (for $E=$ 0.8 ). Excessively high acceptor excitation intensities can thus bias results of the FRET estimation method when using simultaneous donor and acceptor excitation and evaluating of the FRET efficiencies from the donor intensities. Potentially undesirable photochemical side reactions are also an issue (Sinnecker et al. 2005). 
The involvement of triplet states was neglected in this study as it was carried out under steady-state conditions without exclusion of oxygen. In a time resolved experiment, triplet states may also populate to a finite degree and contribute to donor dequenching, inasmuch as the triplet does not generally function as a FRET acceptor. However, this effect would be included in our estimation of the saturation coefficient from the measured acceptor intensity.

Another promising approach would be to employ donor saturation with high power pulsed illumination (JaresErijman and Jovin 2003). Such a protocol would facilitate application in widefield microscopy, also resulting in a faster acquisition protocol than that implemented in the present study. An additional virtue of such a method would be the positional independence of the saturation fraction $\alpha$. A potential danger of intensely pulsed widefield excitation is the momentary thermal load which can lead to photodisruptive processes.

The new method of saturation FRET demonstrated experimentally here for the first time is an additional and promising technique in the category of acceptor depletion techniques for FRET estimation (Jares-Erijman and Jovin 2003). It has the advantage of being compatible with nearly all commercially available confocal microscopes and provides FRET efficiencies using just two image-frames (with two different excitation scenarios: one where the acceptors are unsaturated and one where the acceptors show a significant amount of saturation, i.e. $>10 \%$ ). Thus, the method can yield a result in $0.1 \mathrm{~s}$ and is repeatable. The method yields the information required for assessing systems with low binding efficiencies of the moieties bearing the donor and acceptor, given the existence of the necessary spectral data. It should be possible to differentiate between populations of non-binding and binding FRET pairs using all available spectral characteristics.

Acknowledgments The authors wish to thank Mustapha Faroudi, Victor Tybulewicz and Kate Sullivan (NIMR, London) for the kind provision of the Leica SP2 microscope and their help. We also thank Elena Kardash for the supply of the transfected CHO cells and Klaus Suhling (King's, London) for proof reading the manuscript. The CKAR construct was kindly provided by Alexandra C. Newton. This research was financed by DFG Grant HE 3492/2-1, SPP 1128.

Open Access This article is distributed under the terms of the Creative Commons Attribution Noncommercial License which permits any noncommercial use, distribution, and reproduction in any medium, provided the original author(s) and source are credited.

\section{References}

Allen C, Herman B, Granger N (1992) Fura-2 measurement of cytosolic free $\mathrm{Ca}^{2+}$ concentration in corpus allatum cells of larval Manduca sexta. J Exp Biol 166:253-266
Andrews DL, Demidov AA (1999) Resonance energy transfer. Wiley, Chicester

Bastiaens PIH, Jovin TM (1998) Fluorescence resonance energy transfer microscopy. In: Celis JE (ed) Cell biology: a laboratory handbook, vol 3. Academic Press, New York, pp 136-146

Berney C, Danuser G (2003) FRET or no FRET: a quantitative comparison. Biophys J 84:3992-4010

Beutler M, Wiltshire KH, Meyer B, Moldaenke C, Lüring C, Meyerhöfer $M$ et al (2002) A fluorometric method for the differentation of algal populations in vivo and in situ. Photosynth Res 72:39-53. doi:10.1023/A:1016026607048

Biskup C, Zimmer T, Benndorf K (2004) FRET between cardiac $\mathrm{Na}+$ channel subunits measured with a confocal microscope and a streak camera. Nat Biotechnol 22:220-224. doi:10.1038/ nbt935

Bunt G, Wouters FS (2004) Visualization of molecular activities inside living cells with fluorescent labels International Review of Cytology-a survey of cell biology, vol 237, 237, pp 205

Clayton AHA, Hanley QS, Arndt-Jovin DJ, Subramaniam V, Jovin TM (2002) Dynamic fluorescence anisotropy imaging microscopy in the frequency domain (rFLIM). Biophys J 83:1631-1649

Clayton AHA, Hanley QS, Verveer PJ (2004) Graphical representation and multicomponent analysis of single-frequency fluorescence lifetime imaging microscopy data. J Microsc Oxford 213:1-5. doi:10.1111/j.1365-2818.2004.01265.x

Clegg R (1996) Fluorescence resonance energy transfer. In: Wang HF, Herman B (eds) Fluorescence imaging spectroscopy microscopy. Wiley, New York, pp 179-252

Clegg RM (2006) The history of FRET: from conception through the labors of birth. In: Lakowicz JR, Geddes CD (eds) Reviews in fluorescence, vol 3. Springer, New York, pp 1-45

Colyer R, Lee C, Gratton E (2007) A novel fluorescence lifetime imaging system that optimizes photon efficiency. Microsc Res Tech 71:201-213

Davis LM, Shen GQ, Ball DA (2005) Saturation effects in fluorescence correlation spectroscopy. In: Periasamy A, Peter TC (eds) Multiphoton microscopy in the biomedical sciences V, vol 5700. SPIE, Bellingham, pp 128-137

Digman MA, Caiolfa VR, Zamai M, Gratton E (2008) The phasor approach to fluorescence lifetime imaging analysis. Biophys $\mathbf{J}$ 94:L14-L16. doi:10.1529/biophysj.107.120154

Elangovan M, Wallrabe H, Chen Y, Day RN, Barroso M, Periasamy A (2003) Characterization of one- and two-photon excitation fluorescence resonance energy transfer microscopy. Methods 29:58-73. doi:10.1016/S1046-2023(02)00283-9

Förster T (1948) Intermolecular energy migration and fluorescence. Ann Phys Leipzig 2:55-75. doi:10.1002/andp.19484370105

Gu Y, Di L, Kelsell DP, Zicha D (2004) Ouantitative fluorescence resonance energy transfer (FRET) measurement with acceptor photobleaching and spectral unmixing. J Microsc Oxford 215:162-173. doi:10.1111/j.0022-2720.2004.01365.x

Hanley QS, Arndt-Jovin DJ, Jovin TM (2002) Spectrally resolved fluorescence lifetime imaging microscopy. Appl Spectrosc 56:155-166. doi:10.1366/0003702021954610

Hanninen PE, Lehtela L, Hell SW (1996) Two- and multiphoton excitation of conjugate-dyes using a continuous wave laser. Opt Commun 130:29-33. doi:10.1016/0030-4018(96)00189-7

Hoppe A, Christensen K, Swanson JA (2002) Fluorescence resonance energy transfer-based stoichiometry in living cells. Biophys $\mathrm{J}$ 83:3652-3664

Hoppe A, Shorte SL, Swanson JA, Heintzmann R (2008) D-FRET reconstruction microscopy for analysis of dynamic molecular interactions in live cells. Biophys J 95:400-418

Itoh RE, Kurokawa K, Ohba Y, Yoshizaki H, Mochizuki N, Matsuda $M$ (2002) Activation of Rac and CDc42 video imaged by fluorescent resonance energy transfer-based single-molecule 
probes in the membrane of living cells. Mol Cell Biol 22:65826591

Jares-Erijman EA, Jovin TM (2003) FRET imaging. Nat Biotechnol 21:1387-1395. doi:10.1038/nbt896

Jares-Erijman EA, Jovin TM (2006) Imaging molecular interactions in living cells by FRET microscopy. Curr Opin Chem Biol 10:409-416. doi:10.1016/j.cbpa.2006.08.021

Kremers G, Goedhart J, vanden Heuvel D, Gerritsen H, Gadella T (2007) Improved green and blue fluorescent proteins for expression in bacteria and mammalian cells. Biochemistry 12:3775-3783. doi: $10.1021 / \mathrm{bi} 0622874$

Lakowicz JR (2006) Principles of fluorescence spectroscopy, 3rd edn. Springer, New York

Marriott G, Parker I (2003) Biophotonics, part A and B methods in enzymology. Academic Press, San Diego

van der Meer BW (1994) Resonance energy transfer: theory and data. Wiley, New York

Molecular P (2004) Alexa fluor spanning the visible and infrared spectrum.

http://probes.invitrogen.com/handbook/sections/0103.html

Neher R, Neher E (2004) Optimizing imaging parameters for the separation of multiple labels in a fluorescence image. J Microsc Oxford 213:46-62. doi:10.1111/j.1365-2818.2004.01262.x

$\mathrm{Ng} \mathrm{T}$, Squire A, Hansra G, Bornancin F, Prevostel C, Hanby A et al (1999) Imaging protein kinase $C$ alpha activation in cells. Science 283:2085-2089. doi:10.1126/science.283.5410.2085

Perrin J (1927) CR Acad Sci. (Paris) 184:1097

Peter M, Ameer-Beg SM, Hughes MKY, Keppler MD, Prag S, Marsh $M$ et al (2005) Multiphoton-FLIM quantification of the EGFPmRFP1 FRET pair for localization of membrane receptor-kinase interactions. Biophys J 88:1224-1237. doi:10.1529/biophysj. 104.050153

Redford G, Clegg R (2005) Polar plot representation for frequency domain analysis of fluorescence lifetimes. J Fluoresc 15:805815. doi:10.1007/s10895-005-2990-8

Schuler B, Lipman EA, Steinbach PJ, Kumke M, Eaton WA (2005) Polyproline and the "spectroscopic ruler" revisited with singlemolecule fluorescence. Proc Natl Acad Sci USA 102:2754-2759. doi:10.1073/pnas.0408164102

Sinnecker D, Voigt P, Hellwig N, Schaefer M (2005) Reversible photobleaching of enhanced green fluorescent proteins. Biochemistry 44:7085-7094
Song LL, Hennink EJ, Young IT, Tanke HJ (1995) Photobleaching Kinetics of Fluorescein in Quantitative Fluorescence Microscopy. Biophys J 68:2588-2600

Song LL, Varma C, Verhoeven JW, Tanke HJ (1996) Influence of the triplet excited state on the photobleaching kinetics of fluorescein in microscopy. Biophys J 70:2959-2968

Stryer L (1978) Fluorescence energy-transfer as a spectroscopic ruler. Annu Rev Biochem 47:819-846. doi:10.1146/annurev.bi.47. 070178.004131

Stryer L, Haugland RP (1967) Energy transfer-a spectroscopic ruler. Proc Natl Acad Sci 58:709

$\mathrm{Su}$ WW (2005) Fluorescent proteins as tools to aid protein production. Microb Cell Fact 4:6

Suhling K, French PMW, Phillips D (2005) Time-resolved fluorescence microscopy. Photochem Photobiol Sci 4:13-22

Valentin G, Verheggen C, Piolot T, Neel H, Coppey-Moisan M, Bertrand E (2005) Photoconversion of YFP into a CFP-like species during acceptor photobleaching FRET experiments. Nat Methods 2:801. doi:10.1038/nmeth1105-801

Violin JD, Zhang J, Tsien RY, Newton AC (2003) A genetically encoded fluorescent reporter reveals oscillatory phosphorylation by protein kinase C. J Cell Biol 161:899-909

Widengren J, Rigler R (1996) Mechanisms of photobleaching investigated by fluorescence correlation spectroscopy. Bioimaging 4:149-157. doi:10.1002/1361-6374(199609)4:3<149::AIDBIO5 > 3.3.CO;2-4

Widengren J, Mets U, Rigler R (1995) Fluorescence correlation spectroscopy of triplet-states in solution-a theoretical and experimental study. J Phys Chem 99:13368-13379. doi:10.1021 /j100036a009

Wlodarczyk J, Woehler A, Kobe F, Ponimaskin E, Zeug A, Neher E (2008) Analysis of FRET-signals in the presence of free donors and acceptors. Biophys J 94:986-1000. doi:10.1529/biophysj. 107.111773

Zimmermann T, Rietdorf J, Girod A, Georget V, Pepperkok R (2002) Spectral imaging and linear un-mixing enables improved FRET efficiency with a novel GFP2-YFP FRET pair. FEBS Lett 531:245-249. doi:10.1016/S0014-5793(02)03508-1 